\title{
Viewing-position effects in the Stroop task: Initial fixation position modulates Stroop effects in fully colored words
}

\author{
Patrick Perret and Stéphanie Ducrot \\ University of Provence, Aix-en-Provence, France, \\ Centre National de la Recherche Scientifique, Aix-en-Provence, France, \\ and Centre PsyClé, Aix-en-Provence, France
}

\begin{abstract}
In two experiments that we conducted with adult (Experiment 1) and child (Experiment 2) participants, we experimentally controlled the eyes' first fixation in the word using a variable viewing-position technique in a classical all-letter-coloring Stroop procedure. We explored the impact of initial-fixation position (optimal viewing position [OVP] vs. end of the word) on the magnitude of Stroop effects (both interference and facilitation). The results showed that both interference and facilitation effects were reduced when the first fixation was located at the end of the word rather than at the OVP. These data make a new contribution to the study of the role of low-level processes in Stroop effects and add support to the growing body of research indicating that oculomotor processes can act as moderators of cognitive processes in the determination of Stroop effects.
\end{abstract}

In the Stroop task (Stroop, 1935), participants are instructed to name the color of the ink in which stimuli are presented. A Stroop experiment classically compares three conditions. In the incongruent condition, the stimulus is a color word that is printed in a different color from the one it designates (e.g., the word BLUE printed in green). In the congruent condition, the word and the ink color correspond. Finally, the control condition consists of neutral words or nonwords and provides a baseline for assessing the accuracy and speed with which participants carry out the basic task of naming the ink color. Comparisons of response times (RTs) in these three conditions typically reveal an interference effect (longer RTs in the incongruent condition than in the control condition) and a facilitation effect (shorter RTs in the congruent condition than in the control condition). Interference in the incongruent condition stems from the differential automaticity of the two processes that conflict on those items: reading the word versus naming the ink color (MacLeod \& MacDonald, 2000).

The mere existence of the Stroop effect is often cited as empirical evidence for the automaticity of reading, which is thought to occur without the possibility of being controlled. This view is supported by many studies indicating that Stroop effects persist in experimental conditions that should help participants ignore the meaning of the word (see, e.g., Lachter, Ruthruff, Lien, \& McCann, 2008; see also Lien, Ruthruff, Kouchi, \& Lachter, 2010, for a discussion). Participants thus seem to process printed words in the same way, regardless of whether they are informative for the task at hand. In two recent studies, researchers further documented this phenomenon in a new way that took into account the oculomotor dimension of the task, which has been largely neglected up to now in the vast literature on the Stroop effect. In the first study, Hodgson, Parris, Gregory, and Jarvis (2009) investigated the effect of linguistic stimuli on eye-movement programming, using a modified version of the Stroop task that required a saccadic response rather than a verbal or buttonpress response. The participants' task was to respond by looking toward one of the four color patches that matched the "ink" color of a centrally presented word and to ignore the word's meaning. Their results demonstrated that saccadeprogramming processes were affected by the word's meaning even when the word form was irrelevant to task performance. In addition, they observed very short intersaccade intervals between initial errors and subsequent corrective saccades, thus suggesting that saccadic responses were programmed in parallel to two goals defined by both the cue word's meaning and color. The authors concluded that written-word cues could "capture" saccadic behavior in a manner similar to that found for peripheral visual onsets, in a task for which the semantic content of word stimuli must be ignored to effectively perform the task. In the second study, Smilek, Solman, Murawski, and Carriere (2009) used an eyetracking device to record participants' eye movements during the processing of Stroop material. The results indicated that the first eye fixations were systematically biased to fixate a particular position in the word: the optimal viewing position (OVP). A typical finding regarding the perception of written words by adults 
is that the ease with which printed words are recognized depends on the position at which the eyes initially fixate. Word-recognition performance is maximal slightly to the left of the word's center and decreases on both sides of this OVP (O'Regan, Lévy-Schoen, Pynte, \& Brugaillère, 1984). It has been shown that the typical leftward asymmetry of the viewing-position curve cannot be accounted for in terms of acuity alone, but that it results from the combined influence of several factors - namely, cerebral asymmetries (Brysbaert, Vitu, \& Schroyens, 1996), attentional/perceptual biases ${ }^{1}$ (e.g., Nazir, Ben-Boutayab, Decoppet, Deutsch, \& Frost, 2004; Nazir, Jacobs, \& O'Regan, 1998), and the distribution of information within the printed word in languages such as English and French ${ }^{2}$ (O'Regan et al., 1984; Stevens \& Grainger, 2003). Smilek et al.'s (2009) data indicated that, despite the participants' efforts to prevent themselves from processing the words in the Stroop task, eye-movement patterns (1) closely paralleled those observed during reading and (2) paradoxically favored the meaning-extraction process. This finding suggests that oculomotor behavior might influence Stroop effects: The difficulty for the participants to override their tendency to read could be rooted in their spontaneous bias to fixate a position in the word that facilitates word recognition. A crucial empirical argument in favor of this view is that the Stroop effect can be reduced if the first fixation deviates from the OVP. However, in Smilek et al.'s study, this issue could not be addressed. Because too few fixations fell away from the OVP, the authors could not compare the magnitude of the Stroop effect as a function of eye-fixation position.

In a study investigating the single-letter coloring effect (SLCE), Parris, Sharma, and Weekes (2007) provided the first data documenting the influence of the position at which the eyes initially fixate. The SLCE refers to the fact that when a single letter of a Stroop word is colored, the Stroop effect is reduced (see, e.g., Manwell, Roberts, $\&$ Besner, 2004). Parris et al., who suggested that this phenomenon could interact with the OVP effect, compared the SLCE obtained in four conditions, depending on the position of the colored letter in the word: initial letter, end letter, middle letter, or OVP. The results showed that an SLCE (i.e., the difference between Stroop effects obtained in the "all-letter condition" and the "single-letter condition") was found for all letter positions except the OVP. This finding is in line with the hypothesis that eyefixation position can modulate Stroop effects. However, in this experiment, the influence of the OVP was not studied independently of the single-letter coloring procedure. Manwell et al. argued that coloring a single element provides participants with a cue that helps them select a source of activation and aids selective attention. In contrast with situations in which all of the letters are colored and attention is therefore spread throughout the word, coloring only a single letter could enhance the viewing position's influence on the distribution of spatial attention (Besner \& Stolz, 1999) and keep it focused on the single, colored letter. Accordingly, the effect observed by Parris et al. might be specific to the SLCE experimental context and could hardly be generalized to the broader range of classical Stroop studies. Thus, the authors could only conclude that "the OVP can moderate performance in the Stroop task when only one letter is the color carrier" (p. 278, our italics).

\section{The Present Study}

The aim of the present experiments was to explore the effects of initial-fixation location in a Stroop task with fully colored words. In order to achieve this, we needed to override the methodological difficulty that Smilek et al. (2009) encountered: Spontaneous fixations in their eyetracking study did not allow for comparing Stroop effects as a function of initial eye position. To avoid this difficulty, the present study relied on a different experimental paradigm: the variable viewing-position technique, known to elicit a highly stable pattern of performance in skilled readers. ${ }^{3}$ This pattern gives rise to a systematic variation in reading performance as a function of the eye-fixation position in the word: Word recognition performance is best when the eyes fixate slightly to the left of the word's center and decreases when the eyes deviate from this optimal viewing position and move toward the beginning or the end of the letter string (O'Regan et al., 1984). In two experiments conducted with adult (Experiment 1) and child (Experiment 2) participants, we experimentally controlled the eyes' first fixation in the word (at the OVP vs. at the end of the word ${ }^{4}$ ) and explored its impact on Stroop effects (both interference and facilitation) in a classical all-letter-coloring procedure. In these experiments, participants had to identify the color of the ink in which stimuli were printed, in a computerized Stroop experiment. The stimuli were either incongruent (e.g., the word RED printed in green), congruent (e.g., the word RED printed in red), or neutral (rows of colored hash marks). Considering the aforementioned theoretical arguments, our main prediction was that an eye fixation at the OVP would increase the efficiency of word processing. Accordingly, we expected greater interference and facilitation effects in the OVP condition than in the condition in which the initial eye fixation was located at the end of the word.

\section{EXPERIMENT 1}

\section{Method}

Participants. Twenty-nine students from the University of Provence volunteered to participate. Their mean age was 20.1 years. All were native speakers of French and reported normal or correctedto-normal vision.

Design and Stimuli. Two sets of stimuli were used. The stimuli for the congruent and incongruent conditions were the four French color words: bleu, vert, rouge, and jaune (blue, green, red, and yellow, respectively). For the neutral condition, we used strings of hash marks matching the length of the color words used in the experiment-for example, \#\#\#\# for ROUGE (red). Half of the stimuli were fourletter words, and the other half were five-letter words. The stimuli were equally likely to be colored in red (RGB $255,0,0)$, blue (RGB $0,0,255)$, green (RGB 5,220,75), or yellow (RGB 255,255,0) and appeared on a black background. Each stimulus was divided into five equally wide zones (i.e., 0.8 letters wide for four-letter words and 1.0 letters wide for five-letter words). The stimuli were presented in such 
a way that participants initially fixated either the center of the OVP zone (just to the left of the word's center), or the center of the rightmost zone (hereafter called positions P3 and P5). Across all participants, each stimulus was seen from both of these fixation positions. In Experiment 1, we thus manipulated trial congruency (neutral vs. congruent vs. incongruent) and initial fixation position (P3 vs. P5) in a $3 \times 2$ factorial design. All of the factors were manipulated within participants. There were 12 trials at each congruency level and at each fixation position, for a total of 72 experimental trials.

Apparatus and Procedure. Participants were tested individually. The stimulus presentation took place on a 17-in. color monitor (background luminance of $0.5 \mathrm{~cd} / \mathrm{m}^{2}$ ) that was connected to a Pentium III laptop computer running on DMDX software, Version 2.9.01 (Forster \& Forster, 2003). The stimuli were displayed in uppercase colored letters on a black background in 24-point Courier New font (luminance of $25 \mathrm{~cd} / \mathrm{m}^{2}$ ), with a $640 \times 480$ resolution. Participants were seated $60 \mathrm{~cm}$ from the screen. At this distance, one letter sustained $1^{\circ}$ of visual angle. Each trial consisted of the following sequence of events (see Figure 1). At the beginning of a trial, participants had to fixate the cross displayed in the middle of the screen and not move their eyes. The importance of continuing to focus on this point was stressed repeatedly. Then, $500 \mathrm{msec}$ later, the fixation point was replaced by a stimulus that remained on the screen until the participant responded. The stimulus was displayed off-center - that is, shifted to the side with respect to the fixation point, depending on the position condition. In the P3 condition, stimuli were presented in such a way that the participants initially fixated the center of the OVP zone, located slightly left of center. In the P5 condition, stimuli were presented with their last letters on the central fixation point. The participant's task was to indicate the color in which each item was printed, as quickly and accurately as possible, by pressing one of four keys on a buttonbox. Once the participant had responded, the screen was cleared and a new trial began after a 1,000-msec delay. The order of the trials was completely randomized, with the constraint that none of the three conditions could occur more than three times in succession. Short participantcontrolled breaks were allowed after 36 trials. A 12-item training phase was held at the beginning of each session, followed by a single experimental block of 72 trials.

\section{Results and Discussion}

Only RT data from correct responses were analyzed. The error data produced no effects of interest and showed no signs of a speed-accuracy trade-off. Before any statistical analyses were conducted, response latency means and standard deviations on correct responses were calculated for each participant in each experimental condition. Any response that was more than $3 S D$ s above the participant mean was removed (see Long \& Prat, 2002, and Berent \& Marom, 2005, for an identical trimming procedure). Outliers accounted for fewer than $1 \%$ of the responses. We assessed the Stroop interference effect by comparing the incongruent condition with the neutral condition, as Besner, Stolz, and Boutilier (1997) did. The RT data were input into a 3 (congruence: congruent vs. neutral vs. incongruent) $\times 2$ (initial fixation position: OVP, P3 vs . word-end, P5) repeated measures ANOVA. The results of Experiment 1 are summarized in Table 1.

These analyses yielded a significant effect of trial congruency in the latency data $[F(2,56)=22.0301$, $p<.0001]$, with RTs that were $40 \mathrm{msec}$ longer in the incongruent-word condition $(695 \mathrm{msec})$ than in the control condition $(655 \mathrm{msec})$ and in the congruent condition (645 msec). Pairwise comparisons indicated a Stroopinterference effect: Responses were significantly slower in the incongruent condition than in the neutral condition $[F(1,56)=23.68, p<.001]$. Conversely, responses were faster in the congruent condition than in the neutral condition, but the difference did not reach significance $[F(1,56)=1.8, p>.10]$.

Responses on P5 trials $(665 \mathrm{msec})$ did not differ significantly $(F<1)$ from responses on $\mathrm{P} 3$ trials $(667 \mathrm{msec})$. The end-fixation position (P5) did not reliably facilitate color identification. However, there was a stronger Stroop effect in condition P3 than in condition P5, and this produced a significant interaction between fixation position and trial congruency $[F(2,56)=10.053, p<.001]$. Pairwise comparisons revealed a significant difference between the congruent and control conditions in $\mathrm{P} 3[F(1,56)=$ $4.05, p<.05]$, but not in $\mathrm{P} 5(F<1)$. Conversely, the difference between the incongruent and control conditions
P3 Condition

Fixation
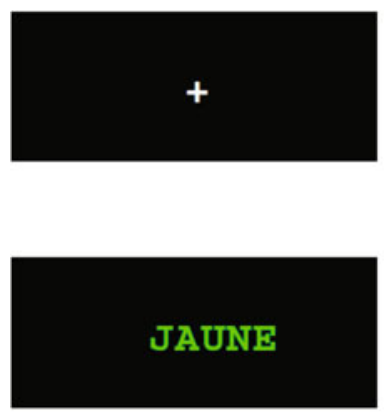

Target Word

(congruent, incongruent, or neutral)
P5 Condition
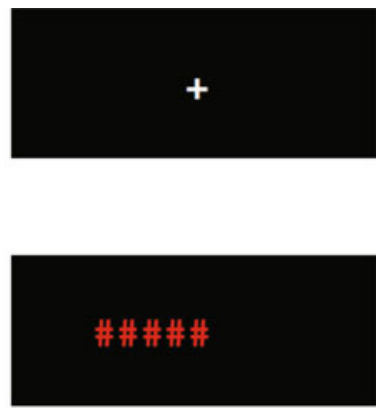

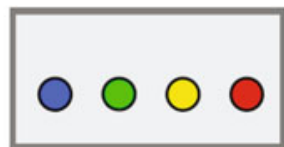

Figure 1. Depiction of the procedure used. 
Table 1

Response Times (RTs) As a Function of Trial Congruency and Initial-Fixation Position

\begin{tabular}{lccccc}
\hline & \multicolumn{3}{c}{ P3 } & & \multicolumn{2}{c}{ P5 } \\
\cline { 2 - 3 } \cline { 5 - 6 } Condition & RT & $S D$ & & RT & $S D$ \\
\hline Congruent & 631.99 & 90.25 & & 658.78 & 92.34 \\
Control & 651.38 & 69.75 & & 659.51 & 86.36 \\
Incongruent & 713.18 & 87.48 & & 676.15 & 83.20 \\
\hline
\end{tabular}

was significant in the $\mathrm{P} 3$ condition $[F(1,56)=72.9, p<$ $.0001]$, but did not reach significance in the $\mathrm{P} 5$ condition $[F(1,56)=2.8, p=.10]$, thus resulting in weaker interference and facilitation effects for condition P5 than for condition $\mathrm{P} 3$.

The results of Experiment 1 are clear-cut. This is the first study showing that Stroop effects can be modulated by the eye-position variable when all of the letters in the word act as the color carrier: Whereas traditional interference and facilitation effects were observed in condition P3, both disappeared in condition P5. The use of the variable viewing-position technique allowed us to successfully address an unresolved issue in previous eyetracking studies. Indeed, demonstrating that the eye position actually affects the magnitude of interference constitutes a critical argument in favor of Smilek et al.'s (2009) claim that low-level oculomotor processes mediate performance on the Stroop task.

Given the theoretical implications of these data, Experiment 2 was designed to replicate this result. Furthermore, in order to challenge the robustness of the viewing-position effect, we tested it on a sample of school-age children in whom oculomotor and visuoattentional processes are still developing (Rayner, 1986).

\section{EXPERIMENT 2}

In Experiment 2, we applied the same procedure as in Experiment 1 with a group of fifth graders. In this grade, children have already been reading for 4 years but still exhibit eye-movement behavior that is not fully developed (Ducrot, Lété, Sprenger-Charolles, Pynte, \& Billard, 2003; Rayner, 1986). At this age, French children are old enough for reading automaticity to have reached at least an intermediate stage (see Samuels, LaBerge, \& Bremer, 1978, for English). At the same time, although adult oculomotor strategies are not expected to be fully effective yet, an OVP effect is already observed in word-recognition studies (Aghababian \& Nazir, 2000; Ducrot et al., 2003). The conjunction of these developmental trends thus makes this age range a particularly good one for challenging the robustness of the eye-position effect.

Participants. Fifty-nine fifth graders who were native speakers of French participated in the experiment. They were from two different classes of an elementary school located in Gap, a city in southern France. They were between 9 and 12 years of age (mean age $=10$ years, 11 months). All of the participants reported normal or corrected-to-normal vision.
}

Materials, Apparatus, and Procedure. The stimuli, apparatus, and procedure were the same as in Experiment 1.

\section{Results and Discussion}

The results were analyzed as in Experiment $1^{5}$ and are summarized in Table 2.

As in Experiment 1, trial congruency generated a significant main effect $[F(2,116)=13.028, p<.0001]$, reflecting the fact that RTs were longer in the incongruent condition $(915 \mathrm{msec})$ than in the control condition $(890 \mathrm{msec})$ and in the congruent condition $(867 \mathrm{msec})$. Pairwise comparisons indicated a Stroop facilitation effect: Responses were faster in the congruent condition than in the neutral condition $[F(1,116)=6.257, p=.013]$. There was also a Stroop interference effect: Responses were significantly slower in the incongruent condition than in the neutral condition $[F(1,116)=6.775, p=.010]$.

We found no differences between the RTs observed on P3 $(888 \mathrm{msec})$ and P5 $(893 \mathrm{msec})$ trials $(F<1)$. But there was a significant trial congruency $\times$ fixation position interaction $[F(2,116)=7.426, p=.0009]$, with a stronger Stroop effect in P3 than in P5. Pairwise comparisons revealed a significant difference between the congruent and control conditions in $\mathrm{P} 3[F(1,116)=15.97, p=.0003]$ but not in $\mathrm{P} 5(F<1)$. Conversely, the difference between the incongruent and control conditions was significant in the $\mathrm{P} 3$ condition $[F(1,116)=8.22, p=.005]$ but not in the $\mathrm{P} 5$ condition $[F(1,116)=1.931, p=.16]$, thus resulting in weaker interference and facilitation effects for condition P5 than for condition P3.

As can be seen in Table 2, we still observed the standard Stroop effect, and it was of about the same magnitude as in Experiment 1 ( $24 \mathrm{msec}$ vs. $10 \mathrm{msec}$ for the facilitation effect and $25 \mathrm{msec}$ vs. $39 \mathrm{msec}$ for the interference effect in Experiments 2 and 1, respectively). Stroop dilution effects that were comparable to those observed with adults were evident by the end of the fourth year of reading instruction.

\section{GENERAL DISCUSSION}

Our present experiments provided a new contribution to the study of the role of low-level processes in Stroop effects. The results of Experiment 1 showed that the first eye fixation in a word clearly modulates the Stroop phenomenon: Whereas traditional interference and facilitation effects were observed in the OVP condition, both were found to be significantly reduced when participants were experimentally led to focus on the last letter of Stroop

Table 2

Response Times (RTs) As a Function of Trial Congruency and Initial-Fixation Position

\begin{tabular}{lccccc}
\hline & \multicolumn{3}{c}{ P3 } & & \multicolumn{2}{c}{ P5 } \\
\cline { 2 - 3 } \cline { 5 - 6 } Condition & RT & $S D$ & & RT & $S D$ \\
\hline Congruent & 846.47 & 130.87 & & 886.64 & 138.20 \\
Control & 891.99 & 132.57 & & 888.56 & 129.71 \\
Incongruent & 925.56 & 154.60 & & 904.61 & 139.49 \\
\hline
\end{tabular}


stimuli. The replication of the viewing-position effect with a sample of fifth graders in Experiment 2 further documented both the generalizability and the robustness of the effect: It can be observed at the earliest stages of automaticity and also when OVP effects in reading are stabilizing. These findings extend previous demonstrations of the importance of the OVP in single-word processing (O'Regan \& Jacobs, 1992; O'Regan et al., 1984; see also Ducrot \& Pynte, 2002). They also extend the results of Parris et al. (2007), which revealed an interaction between OVP and SLCE. Demonstrating that a viewing-position effect is observed independently of the single-letter-coloring procedure clearly widens the scope of Stroop studies, which should hereafter take the oculomotor dimension of the task into account.

The use of the variable viewing-position technique in our present study allowed us to confirm that the interference effect is weaker if participants are led to move their eyes away from the OVP. Our interpretation of these data is that the eye-position variable may be responsible for a shift in the way in which interference is resisted. The replication of the Stroop interference effect in condition P3 is in line with the idea that skilled readers tend to process printed words when they are presented foveally, even if the written word provides no task-relevant information. In this case, resistance to interference must be achieved by suppressing the irrelevant color-word representation before it takes control of responding. Conversely, condition P5 may promote a preventive mode of resistance to interference on incongruent trials by preventing the reading process itself. If letters presented on the right are perceived better than those presented on the left (which is presumably the case for French monolingual participants), then fixating to the right of the word's center (P5) is likely to reduce the overall letter visibility and prevent participants from reading the word. As a consequence, it could reduce the proportion of items for which a time-consuming process of conflict resolution is required, thus resulting in a smaller interference effect. A complementary account could be derived from a standard finding in the literature on visual word recognition: Words presented to the right visual field are easier to recognize than words presented to the left visual field (Lindell \& Nicholls, 2003). A possible explanation for this asymmetry is that fixations on the left half of the word are less "damaging" than fixations on the right half, because attention can be allocated more rapidly to the right than to the left in people who are trained to read in that direction (see Ducrot \& Grainger, 2007). Note that in condition P5 of our experiment, the first fixation was on the last letter of the word, which was thus displayed in the left visual field.

Taken together, the results of the present study add support to Smilek et al.'s (2009) suggestion that experimental variations in the Stroop effect may partially rest on low-level oculomotor processes: Stroop effects are expected to decrease in conditions that favor eye fixations away from the OVP in such a way that word-recognition processes operate much less efficiently (e.g., the eyes are prevented from fixating - or are drawn away from - the OVP). Insofar as this factor is now known to affect both children's and adults' resistance to interference, it should also be seriously considered in future interpretations of individual variability in Stroop performance. In this perspective, future investigations should assess the extent to which individual differences in Stroop performance could be associated with differences in eye-movement patterns. In the introduction of the present article, we suggested that reading involves managing eye movements in order to optimize information extraction. The present data indicate that this also holds true for reading prevention, which is the participant's goal in a Stroop task. Individual eye-movement differences might moderate the respective contributions of prevention and suppression mechanisms, which in turn would affect their performance. To address this issue, two lines of research are presently planned: (1) study the eye-movement patterns of adults with varying degrees of Stroop-task expertise, and (2) explore the impact of dyslexia - which gives rise to specific visualattentional processing (Brysbaert \& Meyers, 1993; Ducrot et al., 2003; Everatt, 1999; Lété \& Ducrot, 2008)—on facilitation and interference effects in the Stroop task.

\section{AUTHOR NOTE}

Partial support for the present research was provided by a grant from the Conseil Régional PACA and the Conseil Général des Bouches du Rhône to S.D. We thank Benjamin Parris, Daniel Smilek, Evan Risko, and Joël Pynte for their helpful comments on previous versions of this article. Correspondence concerning this work can be addressed to P. Perret, Laboratoire PsyCLE, Université de Provence, 29 Av. Robert Schuman, 13621 Aix-en-Provence Cedex 1, France (e-mail: patrick.perret@, univ-provence.fr).

\section{REFERENCES}

Aghababian, V., \& NAZIR, T. (2000). Developing normal reading skills: Aspects of visual processes underlying word recognition. Journal of Experimental Child Psychology, 76, 123-150.

Berent, I., \& Marom, M. (2005). The skeletal structure of printed words: Evidence from the Stroop task. Journal of Experimental Psychology: Human Perception \& Performance, 31, 328-338.

Besner, D., \& Stolz, J. A. (1999). What kind of attention modulates the Stroop effect? Psychonomic Bulletin \& Review, 6, 99-104.

Besner, D., Stolz, J. A., \& Boutilier, C. (1997). The Stroop effect and the myth of automaticity. Psychonomic Bulletin \& Review, 4, 221225

Brysbaert, M., \& Meyers, C. (1993). The optimal viewing position for children with normal and with poor reading abilities. In S. F. Wright \& R. Groner (Eds.), Facets of dyslexia and its remediation (pp. 107123). Amsterdam: Elsevier.

Brysbaert, M., Vitu, F., \& Schroyens, W. (1996). The right visual field advantage and the optimal viewing position: On the relation between foveal and parafoveal word recognition. Neuropsychology, 18, 385-395.

Ducrot, S., \& Grainger, J. (2007). Deployment of spatial attention to words in central and peripheral vision. Perception \& Psychophysics, 69, 578-590.

Ducrot, S., Lété, B., Sprenger-Charolles, L., Pynte, J., \& BilLARD, C. (2003). The optimal viewing position effect in beginning and dyslexic readers. Current Psychology Letters: Behaviour, Brain \& Cognition, 10, 1-10.

Ducrot, S., \& Pynte, J. (2002). What determines the eyes' landing position in words? Perception \& Psychophysics, 64, 1130-1144.

Everatt, J. (1999). Reading and dyslexia: Visual and attentional processes. London: Routledge.

Forster, K. I., \& Forster, J. C. (2003). DMDX: A Windows display program with millisecond accuracy. Behavior Research Methods, Instruments, \& Computers, 35, 116-124. 
Hodgson, T. L., Parris, B., Gregory, N., \& Jarvis, T. (2009). The saccadic Stroop effect: Evidence for involuntary programming of eye movements by linguistic cues. Vision Research, 49, 569-574.

Jordan, T. R., Patching, G. R., \& Milner, A. D. (1998). Central fixations are inadequately controlled by instructions alone: Implications for studying cerebral asymmetry. Quarterly Journal of Experimental Psychology, 51A, 371-391.

Jordan, T. R., Patching, G. R., \& Thomas, S. M. (2003). Assessing the role of hemispheric specialization, serial-position processing, and retinal eccentricity in lateralized word recognition. Cognitive Neuropsychology, 20, 49-71.

Lachter, J., Ruthruff, E., Lien, M.-C., \& McCann, R. S. (2008). Is attention needed for word identification? Evidence from the Stroop paradigm. Psychonomic Bulletin \& Review, 15, 950-955.

LÉté, B., \& Ducrot, S. (2008). Visuo-attentional deficits in dyslexic readers in the Reicher-Wheeler task. Current Psychology Letters: Behaviour, Brain \& Cognition, 24, 25-39.

Lien, M.-C., Ruthruff, E., Kouchi, S., \& Lachter, J. (2010). Even frequent and expected words are not identified without spatial attention. Attention, Perception, \& Psychophysics, 72, 973-988.

Lindell, A. K., \& Nicholls, M. E. R. (2003). Attentional deployment in visual half-field tasks: The effects of cue position on word naming latency. Brain \& Cognition, 53, 273-277.

Long, D. L., \& PRAT, C. S. (2002). Working memory and Stroop interference: An individual differences investigation. Memory \& Cognition, 30, 294-301.

MacLeod, C. M., \& MacDonald, P. A. (2000). Interdimensional interference in the Stroop effect: Uncovering the cognitive and neural anatomy of attention. Trends in Cognitive Sciences, 4, 383-391.

Manwell, L. A., Roberts, M. A., \& Besner, D. (2004). Single letter coloring and spatial cuing eliminates a semantic contribution to the Stroop effect. Psychonomic Bulletin \& Review, 11, 458-462.

NAZIR, T. A. (2003). On hemispheric specialisation and visual field effects in the perception of print: A comment on Jordan, Patching, and Thomas. Cognitive Neuropsychology, 20, 73-80.

Nazir, T. A., Ben-Boutayab, N., Decoppet, N., Deutsch, A., \& Frost, R. (2004). Reading habits, perceptual learning, and the recognition of printed words. Brain \& Language, 88, 294-311.

NAZIR, T. A., JACOBS, A. M., \& O'REgAN, J. K. (1998). Letter legibility and visual word recognition. Memory \& Cognition, 26, 810-821.

O'REgan, J. K., \& JaCOBS, A. M. (1992). Optimal viewing position effect in word recognition: A challenge to current theory. Journal of Experimental Psychology: Human Perception \& Performance, 18, 185-197.

O'Regan, J. K., Lévy-Schoen, A., Pynte, J., \& Brugaillère, B. (1984). Convenient fixation location within isolated words of different length and structure. Journal of Experimental Psychology: Human Perception \& Performance, 10, 250-257.

Parris, B. A., Sharma, D., \& Weekes, B. (2007). An optimal viewing position effect in the Stroop task when only one letter is the color carrier. Experimental Psychology, 54, 273-280.

RAYNER, K. (1979). Eye guidance in reading: Fixation location within words. Perception, 8, 21-30.

RAYNER, K. (1986). Eye movements and the perceptual span in begin- ning and skilled readers. Journal of Experimental Child Psychology, 41, 211-236.

Samuels, S. J., LaBerge, D., \& Bremer, D. (1978). Units of word recognition: Evidence for developmental changes. Journal of Verbal Learning \& Verbal Behavior, 17, 715-720.

Smilek, D., Solman, G. J. F., Murawski, P., \& Carriere, J. S. A (2009). The eyes fixate the optimal viewing position of task-irrelevant words. Psychonomic Bulletin \& Review, 16, 57-61.

Stevens, M., \& Grainger, J. (2003). Letter visibility and the viewing position effect in visual word recognition. Perception \& Psychophysics, 65, 133-151.

STROOP, J. R. (1935). Studies of interference in serial verbal reactions. Journal of Experimental Psychology, 18, 643-661.

\section{NOTES}

1. Note that perceptual biases may arise from perceptual learning (Nazir, Ben-Boutayab, Decoppet, Deutsch, \& Frost, 2004). According to this account, optimal word recognition will be obtained with eye fixations on the location in the word on which the eyes prefer to land (i.e., between the beginning and the middle of the word; Ducrot \& Pynte, 2002; Rayner, 1979).

2. In languages such as English and French, for example, knowing the first letters of a word typically puts constraints on possible word identity more than does knowing the final letters of a word.

3. Note that Jordan, Patching, and Thomas (2003) pointed out the fact that investigating visual-field asymmetries using lateralized stimuli without using an eyetracker to monitor and control fixation location produces substantial amounts of misleading data (see also Jordan, Patching, $\&$ Milner, 1998, for similar conclusions). For our purpose, the most important point to emphasize is that the OVP effect remains identical, even under the stringent testing procedures adopted by Jordan and colleagues. Moreover, as Nazir (2003) pointed out, Jordan et al. (1998) showed, in a study that was explicitly designed to investigate the effect of fixation errors on performance of laterally displayed words, that "noisy" fixations have virtually no effect on word-recognition performance.

4. An initial-letter condition was not included in this experiment because if performance declines when participants are forced to fixate on the outermost letters of the words, the processing cost is larger for fixations on the end letters than for fixations on the beginning letters (giving the OVP curve a J-shape rather than a U-shape). In this experiment, our aim was to oppose the two most extreme conditions: the OVP, where the greatest number of letters can be recognized, and the end of the word, where the word processing cost is maximal.

5. As in Experiment 1, only RT data from correct responses were analyzed (4.01\% errors). The error data produced no effects of interest and showed no signs of a speed-accuracy trade-off. Before any statistical analyses were conducted, response latency means and standard deviations on correct responses were calculated for each participant in each experimental condition. Any response that was more than $3 S D$ s above the participant mean was removed. Outliers accounted for $1.2 \%$ of the responses.

(Manuscript received September 22, 2009; revision accepted for publication February 17, 2010.) 